Physics Vol. 2, No. 6, pp. 293-310, 1966. Physics Publishing Co. Printed in Great Britain.

\title{
HIGH TEMPERATURE SP-BAND SUPERCONDUCTORS
}

T. H. GEballe, B. T. MATthias*, J.P. REMFikA, A. M. ClOGSTON, V. B. COMPTON, J.P. MAITA and H.J. WILLIAMS

\author{
Bell Telephone Laboratories, Murray Hill, New Jersey \\ (Received 27 January 1966 and in revised form \\ 10 February 1966)
}

\begin{abstract}
The superconductivity, low temperature heat capacity and magnetic susceptibility of a number of face-centered cubic nitrides and carbides of niobium have been studied. In agreement with earlier work it is found that the closer the approach to stoichiometry the higher the transition temperature. The values of the electronic heat capacity coefficient and magnetic susceptibility are unusually low and are accounted for by a proposed model in which the carriers are predominantly in spbands. Insofar as its superconducting properties are concerned the most stoichiometric nitride, $N b N_{0.91}$, may be considered as a sort of "stiff lead". A superconducting transition temperature of $18^{\circ} \mathrm{K}$ is found by extrapolation to hypothetical stoichiometric $\mathrm{NbN}$ which reinforces the empirical suggestion that $18^{\circ} \mathrm{K}$ may be an upper limit for the occurrence of superconductivity.
\end{abstract}

\section{Introduction}

THE cubic interstitial carbide and nitride compounds of niobium belong to a group of superconductors which have rather high transition temperatures, and the compounds have, therefore, been of considerable interest since their discovery. The superconductivity of $\mathrm{NbC}$ was reported by Meissner and Franz [1] in 1930 and that of NbN in 1941 by Aschermann and coworkers [2]. Giorgi and coworkers [3] found that the transition temperature of $\mathrm{NbC}$ was extremely dependent upon the carbon concentration of the compound and that it was highest for the nearly-stoichiometric composition $\mathrm{NbC}_{0.977}$. The transition temperature of this compound, which had the maximum carbon content attainable, was $11.1^{\circ} \mathrm{K}$ whereas the compound $\mathrm{NbC}_{0.70}$ was not superconducting above $1.05^{\circ} \mathrm{K}$. In an extensive investigation of the $\mathrm{Nb}-\mathrm{N}$ system Rögener [4] found a maximum transition temperature of $15.63^{\circ} \mathrm{K}$ for $\mathrm{NbN}_{0.96}$.

It has been shown [5] that the transition temperatures of solid solutions of $\mathrm{NbN}$ and $\mathrm{NbC}$ reach a maximum value of $17.8^{\circ} \mathrm{K}$ which is close to the transition temperature found for the cubic A15 $(\beta-W)$-type compounds such as $\mathrm{Nb}_{3} \mathrm{Sn}, \mathrm{Nb}_{3} \mathrm{Al}$, and $\mathrm{V}_{3} \mathrm{Si}$. The fact that a maximum occurs in $\mathrm{NbC}-$ $\mathrm{NbN}$ solid solutions is, however, markedly different from the superconducting behavior of solid

* And at the University of California, La Jolla, California where the work was supported in part by the U.S. Air Force office of Scientific Research. 
solutions in other systems. For example, there is no maximum in the transition temperature as a function of composition in A15-type solid solutions of two compounds with a common component. The unusual result for the $\mathrm{NbC}-\mathrm{NbN}$ solid solution together with the fact that the transition temperature is close to the highest value reported to date for any material prompted the undertaking of the present study. The superconductivity, low-temperature heat capacity, and magnetic susceptibility of niobium nitride, niobium carbide and a solid solution between them was investigated to gain some insight into their superconducting interactions and also to determine the possibility of obtaining materials with transition temperatures above the present maximum of approximately $18^{\circ} \mathrm{K}$.

\section{Experimental Technique}

The niobium metal powder used in the preparation of the compounds was obtained from United Mineral and Chemical Corporation and the following analysis was supplied: $\mathrm{Fe}-0.03 \%, \mathrm{C}-0.07 \%$, $\mathrm{Ta}-0.1 \%, \mathrm{Ti}-0.01 \%, \mathrm{~N}-0.03 \%, 0-0.29 \%, \mathrm{H}-0.001 \%$, and the chlorination residue $-0.04 \%$. The carbon powder used in the preparation of the carbides was obtained from National Carbon Company and the following analysis was supplied for Grade SP-2: Al $-0.4 \mathrm{ppm}, \mathrm{Cu}-0.2 \mathrm{ppm}$, $\mathrm{Fe}-0.1 \mathrm{ppm}$, and $\mathrm{Si}-0.8 \mathrm{ppm}$. The niobium nitride samples were prepared by compacting $\mathbf{- 3 2 5}$ mesh niobium metal powder in a $1.3 \mathrm{~cm}$ steel die at a pressure of about $1.5 \mathrm{kbars}$. The dimensions of the resulting specimen were about $1.3 \mathrm{~cm}$ diameter by $2.5 \mathrm{~cm}$ long. The niobium compact was placed in a wolfram-lined alumina boat which was then wrapped in $0.003 \mathrm{~cm}$ thick zirconium metal foil. This assembly was placed in a high density alumina furnace tube and purified nitrogen was passed through the tube. A slight positive pressure of gas was maintained by partially clampingoff the exit tube. The nitrogen used was purified by passing tank nitrogen through a quartz tube packed with copper gauze and zirconium metal chips heated to $750^{\circ} \mathrm{C}$. The reaction tube temperature was $1450^{\circ} \mathrm{C}$ and a minimum of $60 \mathrm{hr}$ reaction time was required to equilibrate. A variation in the nitrogen content of the samples was obtained by two different methods of cooling from the reaction temperature. A more nearly-stoichiometric compound was obtained by furnace cooling while water quenching resulted in a compound with a somewhat lower nitrogen content. According to the results of chemical analyses, the furnace-cooled sample contained $11.90 \mathrm{wt} . \%$ nitrogen, corresponding to $\mathrm{NbN}_{0.91}$, and the water-quenched sample contained $10.78 \mathrm{wt} . \%$ nitrogen, corresponding to $\mathrm{NbN}_{0.84}$. The $\mathrm{NbN}_{0.91}$ sample also contained $0.34 \mathrm{wt} . \%$ oxygen and $0.01 \mathrm{wt} . \%$ carbon. The $\mathrm{NbN}_{0.84}$ sample was not analyzed for these elements.

The preparation of all but one of the niobium carbide samples was similar to that of the niobium nitride samples. The compacts of intimately mixed niobium and carbon powders were heated to $1700^{\circ} \mathrm{C}$ in a stream of purified helium for at least $60 \mathrm{hr}$ and furnace-cooled. More nearly-stoichiometric compounds were prepared by the addition of 1 to 2 per cent excess carbon which may act as a getter by reacting with any oxide layer on the niobium particles and forming a gaseous product. The niobium carbide containing the least amount of carbon was prepared by reacting the elements in an arc furnace in an argon atmosphere. The niobium rod used for this sample was obtained from the Wah Chang Corporation and the chief impurities as determined by the supplier were: $\mathrm{Fe}<100 \mathrm{ppm}$, Si $<100 \mathrm{ppm}, \mathrm{Ta}<500 \mathrm{ppm}, \mathrm{Ti}<150 \mathrm{ppm}$, and $\mathrm{W}<200 \mathrm{ppm}$. The carbon rod was supplied by National Carbon Company and although a detailed analysis was not available, its purity is probably at least as good as that of the carbon powder.

The carbide-nitride solid solution was prepared by nitriding a mixture of niobium carbide and niobium powders pressed into a compact as described earlier. According to a chemical analysis the sample contained $3.43 \mathrm{wt} . \%$ carbon and $8.98 \mathrm{wt} . \%$ nitrogen, corresponding to the formula $\mathrm{NbC}_{0.30} \mathrm{~N}_{0.70}$. In addition, the sample contained $118 \mathrm{ppm}$ of oxygen. The niobium nitride 
samples containing small amounts of chromium were made by nitriding compacts of intimate mixtures of niobium and chromium metal powders in the described manner.

The composition of the nitride samples and of the carbide-nitride solid solution was determined by chemical analysis and that of the carbide samples was estimated by comparison of their superconducting transition temperatures and lattice constants with those reported in the literature $[3,6]$. The compositions of the niobium-chromium nitride samples are nominal.

A standard volume, $0.016 \mathrm{~cm}^{3}$ of powder of approximately $0.01 \mathrm{~mm}$ diameter particle size, was prepared from each sample and investigated for superconductivity above $0.32^{\circ} \mathrm{K}$ using the resonant-frequency shift circuit of Schawlow and Devlin [7] and the method previously described [8]. The low-temperature heat capacity data were obtained using a pulse calorimeter [9] and the susceptibility measurements were made using a pendulum magnetometer [10]. X-ray diffraction powder photographs of the samples were taken with a Norelco camera of $114.6 \mathrm{~mm}$ diameter and Cu K-radiation.

\section{Experimental Results}

\section{(a) Superconductivity}

The magnetically and calorimetrically determined superconducting transition temperatures of $\mathrm{NbN}_{0.91}, \mathrm{NbN}_{0.84}, \mathrm{NbC}_{0.30} \mathrm{~N}_{0.70}, \mathrm{NbC}_{0.98}$, and $\mathrm{NbC}_{0.75}$ are given in Table 1. The calorimetric transitions are taken as the temperature at which the heat capacity anomaly first appears; the magnetic transitions are taken as the temperature range over which most (i.e. $\widetilde{7} 75 \%$ ) of the diamagnetic screening currents are established. The agreement between the two methods is good indicating homogeneous samples.

\section{(b) Heat capacity}

Previously reported heat capacities of $\mathrm{B} 1$ type $(\mathrm{NaCl})$-compounds in these systems include those of Armstrong [11] for the nitride in the temperature range of 11 to $21^{\circ} \mathrm{K}$ and sandenaw and Storms [12] for the carbides from 8 to $40^{\circ} \mathrm{K}$. However, these authors were primarily interested in the higher-temperature properties and their results at low temperatures are not accurate or extensive enough for the determination of the electronic properties of these compounds. The low temperature heat capacity data for $\mathrm{NbN}_{0.91}, \mathrm{NbC}_{0.30} \mathrm{~N}_{0.70}, \mathrm{NbC}_{0.98}, \mathrm{NbC}_{0.95}, \mathrm{NbC}_{0.75}$ are $\mathrm{shown}$ in Figs. 1, 2, 3 and 4 respectively and the results for all the compounds investigated are summarized in Table 1. The most striking result of the present investigation is the unusually low value of the electronic heat capacity coefficient, $\gamma$. In the figures this is the intercept at $T=0$ of the extrapolated curve of the normal state heat capacity. Despite some uncertainty in the extrapolations, it is obvious that the $\gamma^{\prime} s$ of these compounds are less than half the $\gamma$ value of pure niobium while their superconducting transition temperatures are almost twice that of niobium.

Since many of the conclusions in the present study are based on the low values of $\gamma$, it is worthwhile to discuss the extrapolation procedure in some detail. There are a number of checks which can be made to reduce the uncertainty of the extrapolations and thereby increase the reliability of the $\gamma$ values so obtained. One of these is the requirement of the third law of thermodynamics that the entropy under the normal state curve from $T=0$ to $T_{c}$ be equal to that measured under the superconducting curve over the same temperature range. If one assumes that 
TABLE 1

Superconductivity, heat capacity, and lattice constant data

\begin{tabular}{|c|c|c|c|c|c|c|c|}
\hline \multirow[t]{2}{*}{ Composition } & \multicolumn{2}{|c|}{$T_{c}, \quad\left({ }^{\circ} \mathrm{K}\right)$} & \multirow{2}{*}{$\begin{array}{c}\theta_{D} \\
\left({ }^{\circ} \mathrm{K}\right)\end{array}$} & \multicolumn{2}{|c|}{$\gamma \times 10^{4}$ cal mole -1 deg. $\mathrm{K}^{-2}$} & \multirow{2}{*}{$C_{e s} / \gamma T_{c}$} & \multirow{2}{*}{$\begin{array}{c}a \\
\left(A^{0}\right)\end{array}$} \\
\hline & Mag. & Cal. & & Extrap. & $3^{\text {rd }}$ Law & & \\
\hline $\mathrm{NbN}_{0.91}$ & $15.60-15.06$ & 15.72 & 307 & 6.3 & 6.3 & 3.9 & 4.391 \\
\hline $\mathrm{NbN}_{0.84}$ & $13.51-12.95$ & 13.68 & 331 & 7.2 & 7.2 & 3.5 & 4.383 \\
\hline $\mathrm{NbC}_{0.30} \mathrm{~N}_{0.70}$ & $17.38-16.63$ & 17.38 & 351 & 7.8 & 7.9 & 3.5 & 4.424 \\
\hline $\mathrm{NbC}_{0.98}$ & $11.58-10.05$ & 11.1 & 464 & 6.3 & 6.5 & 2.5 & 4.470 \\
\hline $\mathrm{NbC}_{0.95}$ & $9.14-6.31$ & 7.62 & 492 & 6.2 & 6.1 & & 4.467 \\
\hline $\mathrm{NbC}_{0.75}$ & $1.0-0.32^{a}$ & & 500 & 5.0 & & & $4.447-4.436$ \\
\hline $\mathrm{Nb}_{0.99} \mathrm{Cr}_{0.01} \mathrm{~N}$ & n.o.b & & & & & & 4.387 \\
\hline $\mathrm{Nb}_{0.97} \mathrm{Cr}_{0.03^{\mathrm{N}}}$ & n. $0 .{ }^{b}$ & & & & & & 4.383 \\
\hline
\end{tabular}

a Transition temperature of a nonpowdered sample

$b$ None observed above $0.32 \% \mathrm{~K}$.

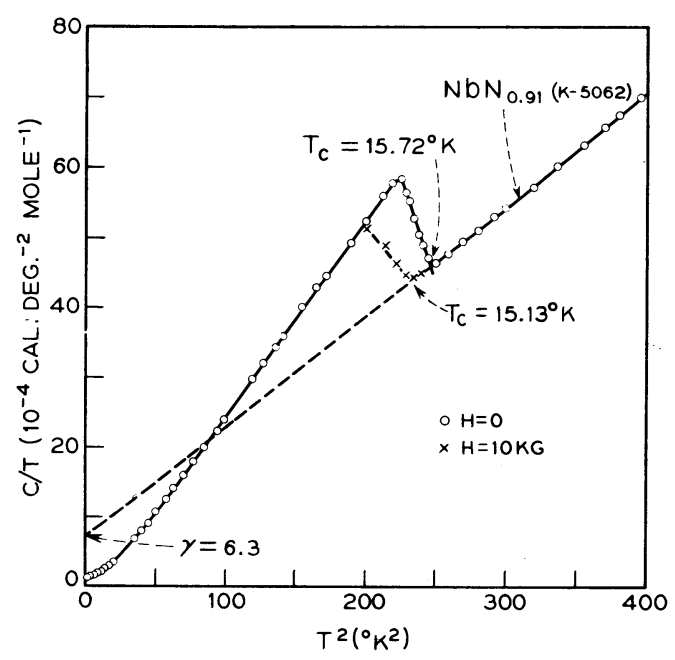

FIGURE 1

Heat capacity of $\mathrm{NbN}_{0.91}$ in zero magnetic field. 


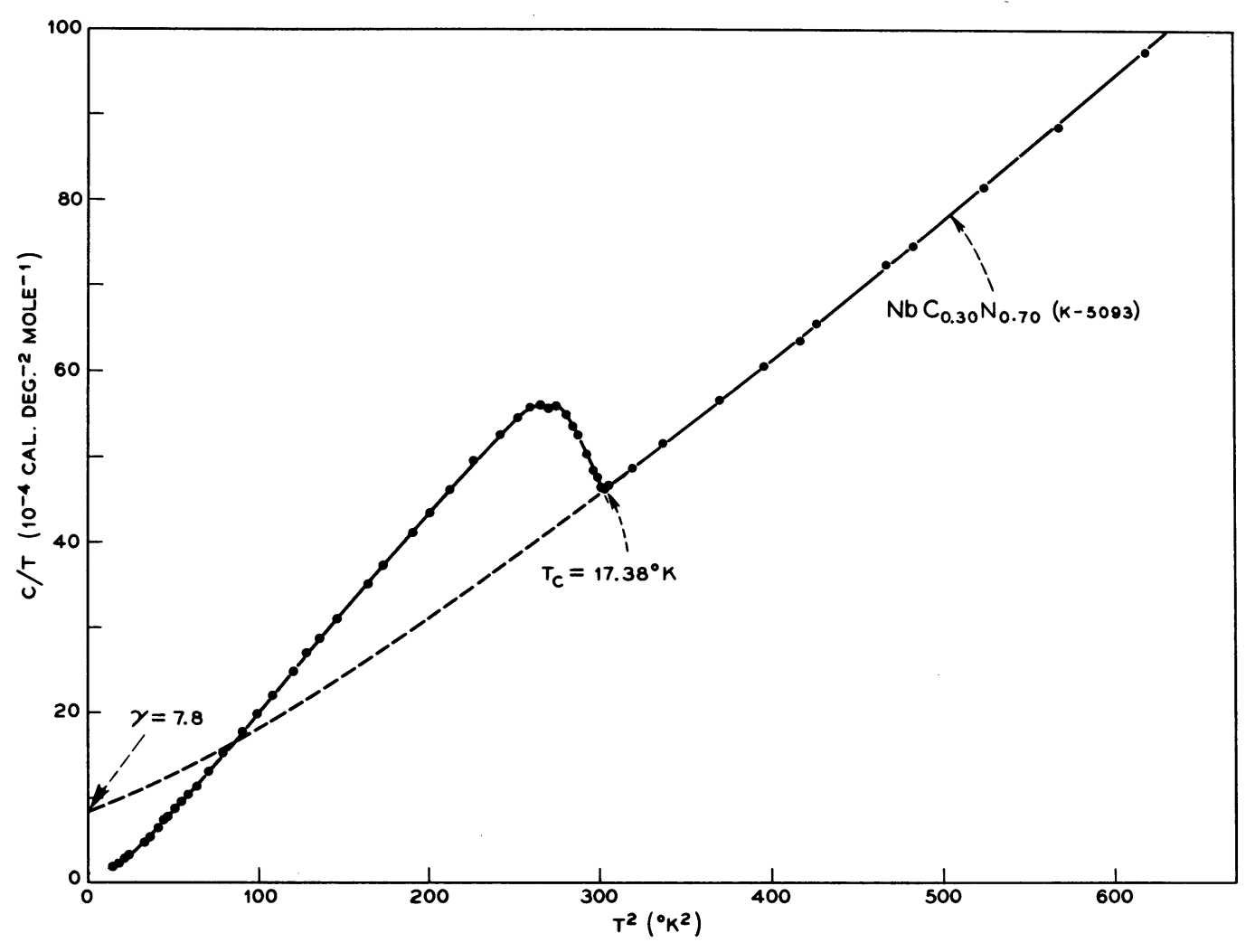

FIGURE 2

Heat capacity of $\mathrm{NbC}_{0.30} \mathrm{~N}_{0.70}$ in zero magnetic field.

the lattice heat capacity is the same in both the normal and the superconducting states, then the $\gamma^{\prime} s$ calculated from the entropy under the superconducting curves should agree with the extrapolated intercept of the normal state curve. As can be seen from Table 1, there is reasonable agreement. An uncertainty in the $\gamma$ value, introduced by the arbitrariness of the curvature of the normal state curve between $T=0$ and $T_{c}$, is estimated to be less than 5 per cent. There is an additional uncertainty, of the order of 3 per cent, in the superconducting entropy of the $\mathrm{NbN}_{0.91}$ and $\mathrm{NbC}_{0.30} \mathrm{~N}_{0.70}$ samples due to an anomalous curvature at the very low temperatures which is shown in Fig. 5. As a result the superconducting curves extrapolate to a finite value at $T=0$, which might be construed as evidence for the presence of a small amount of a second phase. However a magnetic field of $10 \mathrm{kG}$ raises the anomalous heat capacity of $\mathrm{NbC}_{0.30} \mathrm{~N}_{0.70}$ markedly indicating that the extra heat capacity is magnetic in origin. Since the effect does not occur in the niobium carbide samples, which were prepared from the same niobium powder, and since a spectrographic analysis of the $\mathrm{NbN}_{0.91}$ and $\mathrm{NbC}_{0.30} \mathrm{~N}_{0.70}$ samples showed no significant amounts of magnetic impurities, it seems unlikely that the anomalous heat capacity is due to chemical impurities. The entropy associated with this curvature was not included in the calculation of the superconducting entropy since it may not be associated with the superconductivity and therefore, would be present in the normal state. If, however, further investigation shows it should be included, the $\gamma$ values would increase by about 3 per cent.

A two-phase system in which the nonsuperconductor is an insulator could, in principle, lead 
to a false estimate of a low $\gamma$ which would be consistent with the third law requirement. An amount of a second phase appreciable enough to cause a serious underestimate would certainly have been detected in the $\mathrm{X}$-ray diffraction patterns of these samples.

Another check on the consistency of the $\gamma$ values is obtained by comparing them with the observed discontinuity in the heat capacity at $T_{c}$. According to the simple BSC theory $C_{e s} / \gamma T_{c}=$ 2.43. The experimental values as given in Table 1 are somewhat larger and are typical of superconductors with strong phonon-electron coupling such as lead and mercury [13].

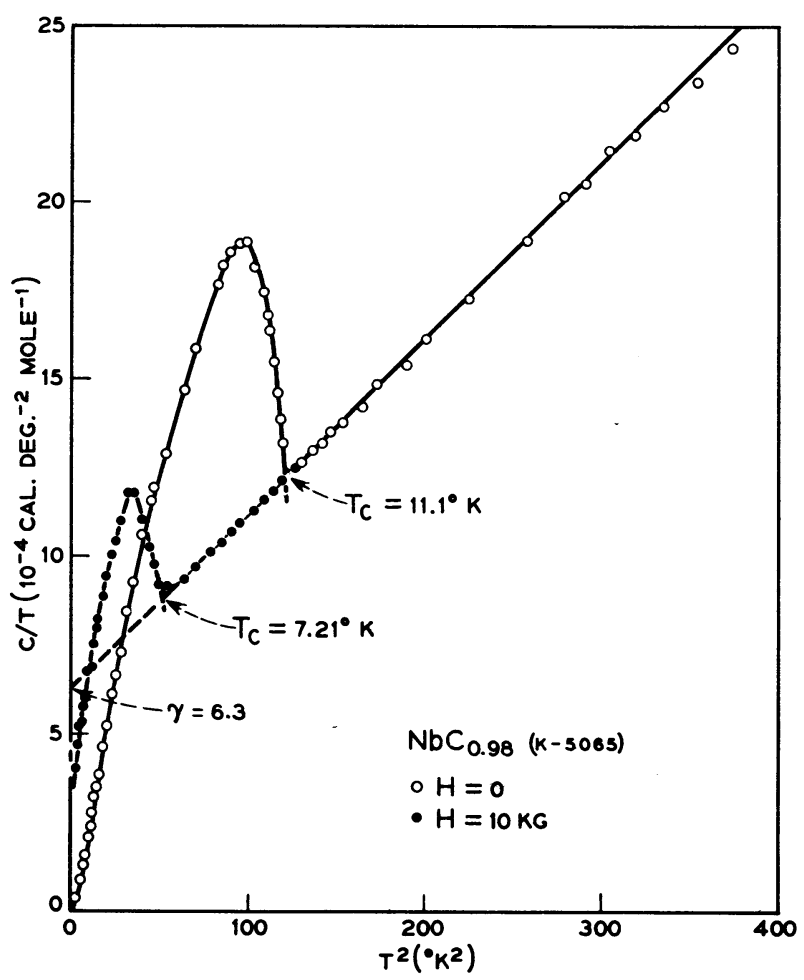

FIGURE 3

Heat capacity of $\mathrm{NbC}_{0.98}$ in zero and $10 \mathrm{~kg}$ magnetic fields.

The application of a magnetic field, which is the usual method for obtaining the $\gamma$ values of superconductors, did not lower the transition temperature of the nitride samples sufficiently for the extrapolation of $C / T$ to $T=0^{\circ} \mathrm{K}$ to be significantly improved. The effect of a $10 \mathrm{kG}$ field on the superconducting transition temperature is shown in Figs. 1, 3 and 4. Since the critical field at $4.2^{\circ} \mathrm{K}$ of some of the samples is over $100 \mathrm{kG}$, this approach was abandoned. Other methods were used to modify the transition temperatures of the compounds in order to check the reliability of the third law extrapolations.

The nearly-stoichiometric Bl type-carbide or nitride has the maximum superconducting transition temperature and deviations from this composition result in a lower transition temperature. The heat capacity data for $\mathrm{NbC}_{0.95}$ and $\mathrm{NbC}_{0.75}$ are shown in Fig. 4 and the results for these compounds and for $\mathrm{NbN}_{0.84}$ are summarized in Table 1 . The $\mathrm{NbC}_{0.95}$ sample shows a trace of superconductivity in zero field at about $11.1^{\circ} \mathrm{K}$ which is the transition temperature of the more nearly-stoichiometric compound $\mathrm{NbC}_{0.98}$, but the major portion of the sample is superconducting 


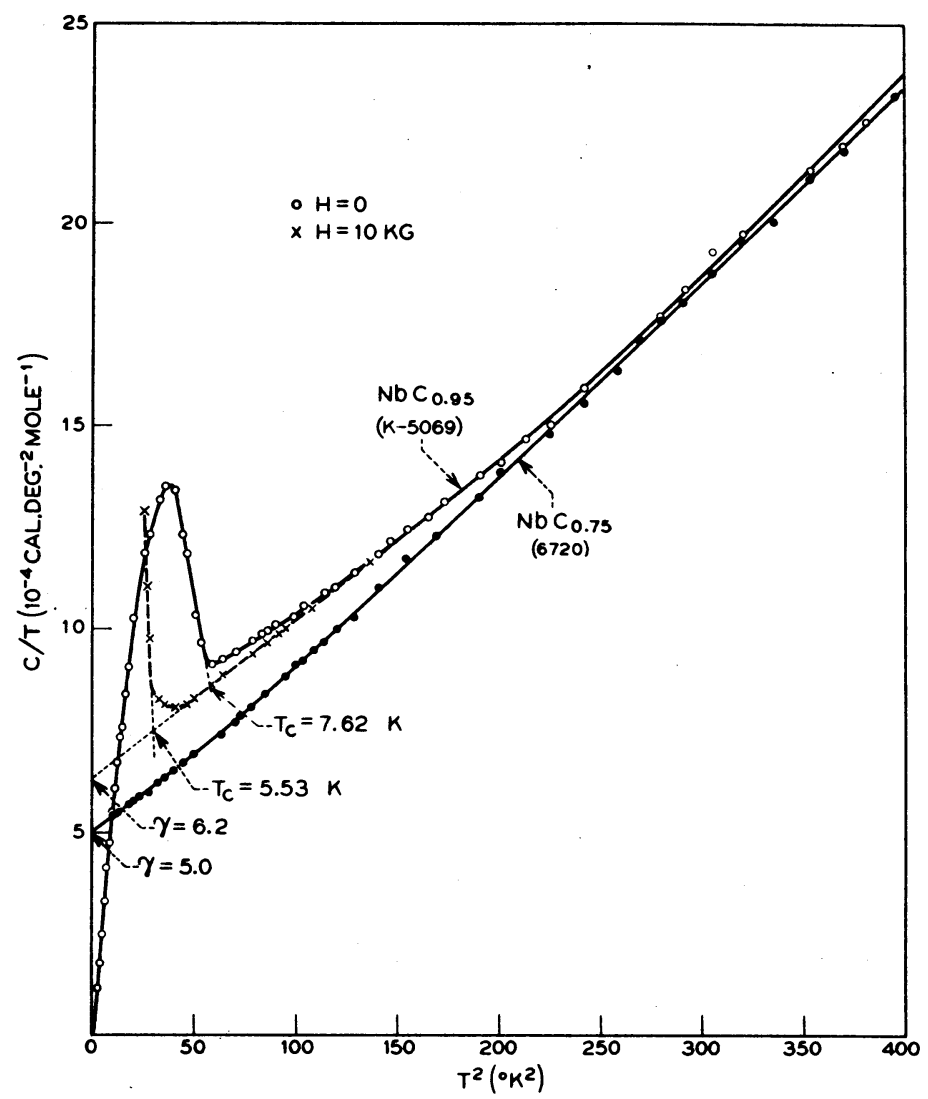

FIGURE 4

Heat capacity of $\mathrm{NbC}_{0.95}$ in zero and $10 \mathrm{~kg}$ magnetic fields and of $\mathrm{NbC}_{0.75}$ in zero magnetic field.

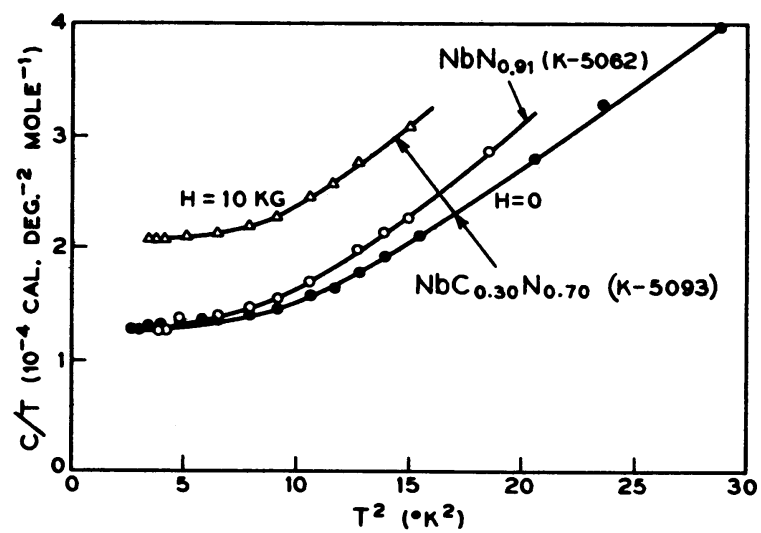

FIGURE 5

Anomalous heat capacity of $\mathrm{NbN}_{0.91}$ and $\mathrm{NbC}_{0.30 \mathrm{~N}_{0.70}}$ 
at $7.52^{\circ} \mathrm{K}$. The application of a $10 \mathrm{~kg}$ field suppresses the small amount of high-temperature superconductivity and lowers the transition temperature of the bulk of the sample to $5.53^{\circ} \mathrm{K}$. The magnetically determined superconducting transition temperature of $\mathrm{NbC}_{0.75}$ was spread out from 1 to $0.32^{\circ} \mathrm{K}$, the lowest temperature measured. An appreciable inhomogeneity undoubtedly is responsible for the broad transition of this arc-melted sample. The transition temperature of $\mathrm{NbN}_{0.84}$ was lowered from $13.68^{\circ} \mathrm{K}$ to $13.11^{\circ} \mathrm{K}$ by the application of a $10 \mathrm{kG}$ field.

An appreciable modification of the transition temperature of niobium nitride was obtained by adding a small amount of chromium. The $\mathrm{Nb}_{0.9}{ }_{9} \mathrm{Cr}_{0.01} \mathrm{~N}$ sample was not superconducting above $0.32^{\circ} \mathrm{K}$ and its heat capacity data are given in Fig. 6. If an allowance is made for the magnetic contribution of chromium, then the extrapolated $\gamma$ value agrees within experimental error with that given in Table 1.

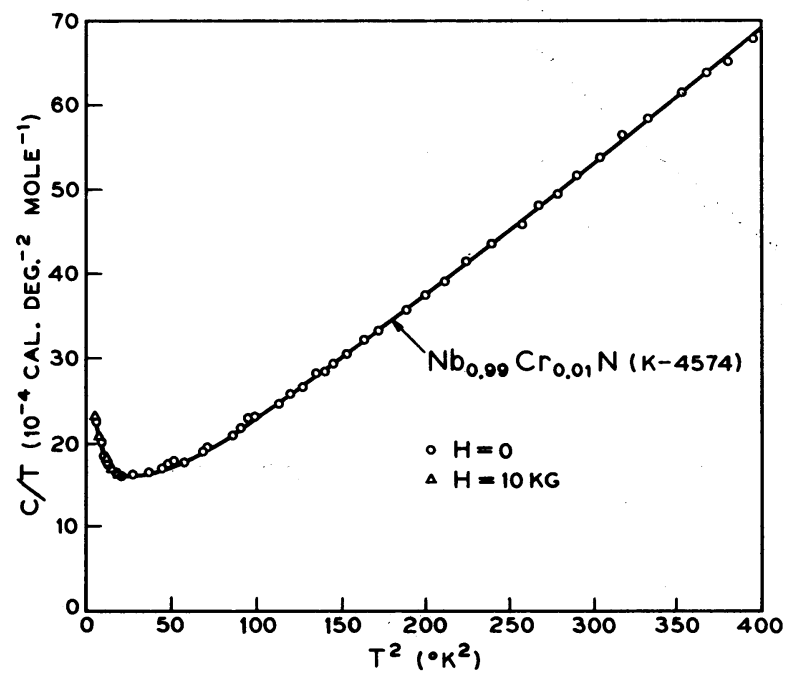

FIGURE 6

Heat capacity of $\mathrm{Nb}_{0.99} \mathrm{Cr}_{0.01^{\mathrm{N}}}$ in zero magnetic field.

\section{(c) Magnetic susceptibility}

The magnetic susceptibility data for $\mathrm{NbN}_{0.91}, \mathrm{NbC}_{0.30} \mathrm{~N}_{0.70}$, and $\mathrm{NbC}_{0.98}$ are given in Figs. 7 , 8 and 9 and the room temperature values are in agreement with those reported by Bittner and coworkers $[14,15]$. As expected from the low $\gamma$ values the susceptibility values are also low. The unusual structure in the $\mathrm{NbN}_{0.91}$ and $\mathrm{NbC}_{0.30} \mathrm{~N}_{0.70}$ curves may be indicative of a phase change at approximately $50^{\circ} \mathrm{K}$. Attempts were made to find a corresponding effect in the heat capacity and in the low-temperature X-ray diffraction data [16], but none was detected. Single crystals of these materials may be required for the clarification of the susceptibility data. It is also possible that the anomalous curvature in the heat capacity curves of these compounds, Fig. 5, is another manifestation of this phenomenon. As can be seen in Fig. 5, the mechanism which gives rise to the extra heat capacity is magnetic field dependent, since a field of $10 \mathrm{~kg}$ causes a marked increase. This is consistent with the paramagnetic behavior observed below the "transition" in the susceptibility curves.

The unusually low values of $\gamma$ and $x$ suggested that there might also be related Knight-shift behavior. Jaccarino and Davis [17] measured the Knight shift of the niobium in the $\mathrm{NbN}_{0.91}$ 


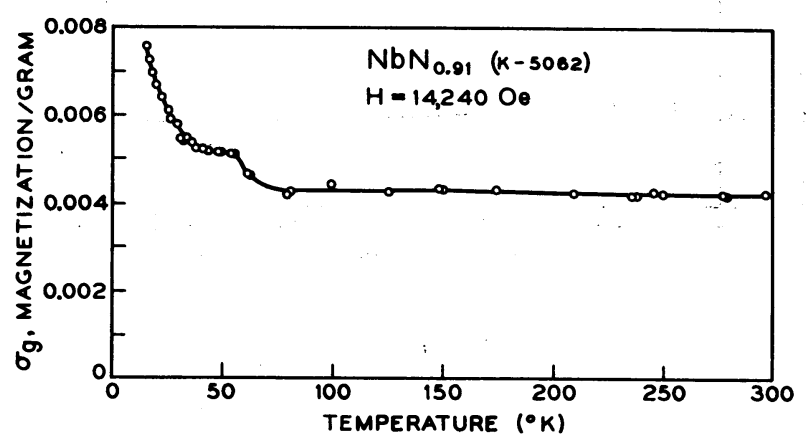

FIGURE 7

Magnetic susceptibility of $\mathrm{NbN}_{0.91}$.

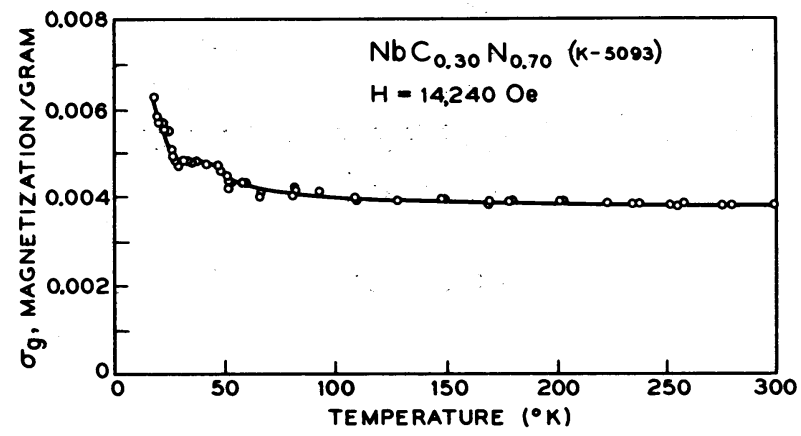

FIGURE 8

Magnetic susceptibility of $\mathrm{NbC}_{0.30} \mathrm{~N}_{0.70}$. 


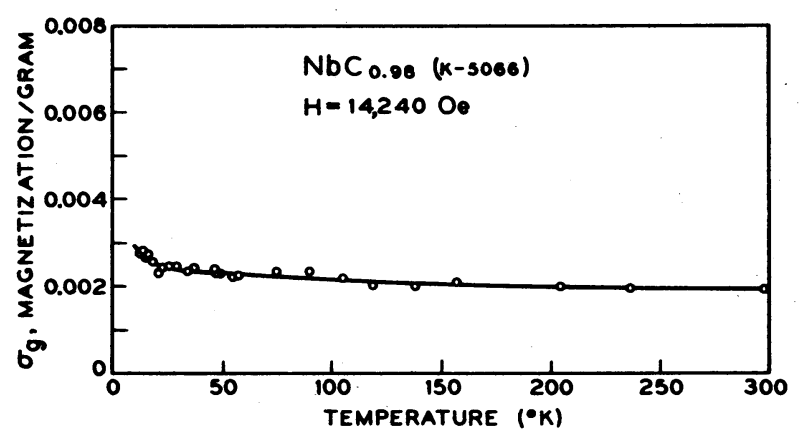

FIGURE 9

Magnetic susceptibility of $\mathrm{NbC}_{0.98}$.

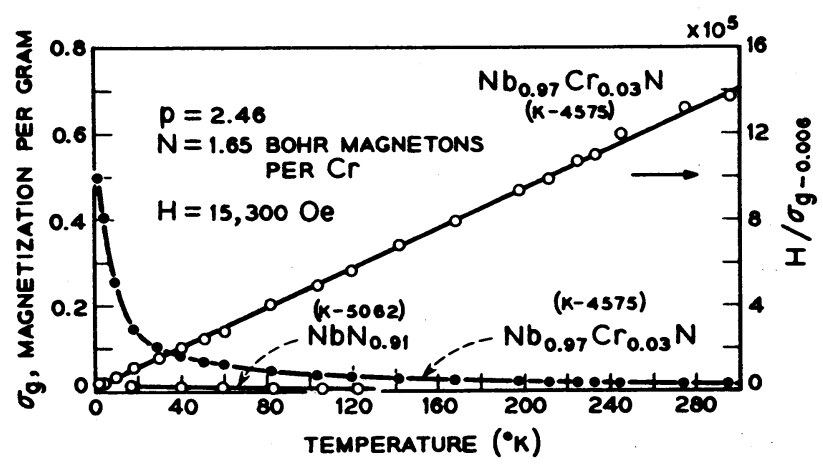

FIGURE 10

Magnetic susceptibility of $\mathrm{Nb}_{0.97} \mathrm{Cr}_{0.03} \mathrm{~N}$. The effective magneton number $p$ comes from the fitting of the data to a Curie-Weiss law. $N$ is the number of Bohr magnetons per Cr atom assuming a $\mathrm{g}$ value of 2.0. 
sample and their results show that the shift is indeed small -10 to 50 times smaller than in niobium metal or in other niobium intermetallic compounds. In addition, there is no significant temperature dependence.

The susceptibility data for $\mathrm{Nb}_{0 .}{ }_{97} \mathrm{Cr}_{0.03} \mathrm{~N}$ are given in Fig. 10. It is of particular interest to note that a dilute solution of chromium in this compound exhibits a localized magnetic moment of $1.65 \mu_{B}$. The same value was also found for the sample containing $1 \% \mathrm{Cr}$ whose heat capacity is shown in Fig. 6.

\section{Discussion and Conclusions}

\section{(a) Superconducting interactions}

If the extrapolations of $C / T$ to $T=0^{\circ} \mathrm{K}$ are accepted, then the surprisingly low values of $\gamma$ must be reconciled with the high superconducting transition temperatures of the compounds. Pessall and Hulm [18] have shown that the superconducting transition temperatures of the interstitial Bl type-compounds are surprisingly insensitive to the substitution of other nonmagnetic $d$-shell metals for the niobium. This result is reminiscent of the superconducting behavior of nontransition element alloys. In fact, a comparison of the interstitial niobium compounds with such superconductors as lead reveals many similarities.

The $\gamma$ value of lead [19] is $7.18 \times 10^{-4} \mathrm{cal} / \mathrm{mol} / \mathrm{deg} \mathrm{K}^{2}$ which is quite close to the values of the interstitial compounds reported in Table 1 . However, the interstitials have considerably higher Debye temperatures, $\theta_{D}$, which accounts for their transition temperature being higher than that of lead. A plot of $\log C_{e s} / \gamma T$ vs. $T_{c} / T$ as shown in Fig. 11 reveals that $\mathrm{NbN}_{0.91}$ and $\mathrm{NbC}_{0.30} \mathrm{~N}_{0.70}$ have the same type of strong-coupling deviation from the simple BCS theory as is observed for lead.

McMillan [20] has shown how to obtain the attractive phonon interaction potential, $V_{p h}$, which enters into the microscopic theory in the strong-coupling case when the heat capacity data are known. This proves to be quite instructive although the procedure must of necessity be rather inexact because of the lack of detailed information of the phonon spectrum and the Coulomb pseudo-potential, $U_{c}$. The input data and the results calculated from McMillan's formula,

$$
\frac{1.45 T_{c}}{\theta}=\exp \left[\frac{-1.04\left(1+N(0) V_{p h}\right)}{N(0)\left[V_{p h}-U_{c}\left(1+0.62 N(0) V_{p h}\right)\right]}\right]
$$

are given in Table 2. A value of 0.1 is assumed for the product of the unrenormalized density of states, $N(0)$, times the pseudo-potential, $U_{c}$, for all the interstitials. The results are not sensitive to this value of $N(0) U_{c}$ since it is much smaller than $N(0) V_{p h}$ which is $\sim 1.0$.

McMillan has found that 0.1 is a reasonable value for lead. Once the value of $N(0) V_{p h}$ is found, $N(0)$ can be obtained from the density of states determined by heat capacity measurements, $N_{\gamma}$. using the expression [21]

$$
N_{\gamma}=N(0)\left(1+N(0) V_{p h}\right)
$$

In Table 2 values for some other high-transition temperature $\beta$-W-type superconductors are also given. These results are more dependent upon $N(0) U_{c}$ because it is much larger relative to 
TABLE 2

Calculation of $N(0)$ and $V_{p h}$ using McMillan's strong-coupling formula

\begin{tabular}{|c|c|c|c|c|c|c|c|}
\hline Compound & $\begin{array}{c}{ }^{N_{\gamma}} \\
\text { Density of states } \\
\text { (per eV per molecule) }\end{array}$ & $\left(\ln \frac{\theta_{D}}{4 T_{c}}\right)^{-1}$ & $N(0) U_{c}$ & $N(0) V_{p h}$ & $N(0)$ & $V_{p h}$ & $\begin{array}{c}\text { Data } \\
\text { References }\end{array}$ \\
\hline $\mathrm{NbN}_{0.91}$ & 0.56 & 0.62 & 0.1 & 0.92 & 0.29 & 3.2 & $a$ \\
\hline $\mathrm{NbN}_{0.84}$ & 0.63 & 0.55 & 0.1 & 0.82 & 0.35 & 2.3 & $a$ \\
\hline $\mathrm{NbC}_{0.30} \mathrm{~N}_{0.70}$ & 0.70 & 0.62 & 0.1 & 0.92 & 0.37 & 2.5 & $a$ \\
\hline $\mathrm{NbC}_{0.98}$ & 0.56 & 0.43 & 0.1 & 0.66 & 0.34 & 1.9 & $a$ \\
\hline $\mathrm{NbC}_{0.95}$ & 0.54 & 0.35 & 0.1 & 0.56 & 0.35 & 1.6 & $a$ \\
\hline $\mathrm{NbC}_{0.75}$ & 0.44 & 0.20 & 0.1 & 0.35 & 0.33 & 1.1 & $a$ \\
\hline $\begin{array}{l}\text { NbN } \\
\text { (hypothetical) }\end{array}$ & 0.5 & 0.75 & 0.1 & 1.1 & 0.24 & 4.6 & \\
\hline $\mathrm{Nb}_{3} \mathrm{Au}$ & $2.3^{*}$ & 0.58 & 0.2 & 1.15 & 1.1 & 1.0 & $b$ \\
\hline $\mathrm{V}_{3} \mathrm{Ge}$ & $2.1 *$ & 0.36 & 0.15 & 0.68 & 1.25 & 0.54 & $c$ \\
\hline $\mathrm{Nb}_{3} \mathrm{Sn}$ & $4.42^{*}$ & 0.67 & 0.20 & 1.30 & 1.96 & 0.66 & $9 ; d$ \\
\hline $\mathbf{v}_{3} \mathbf{S i}$ & $3.85^{*}$ & 0.54 & 0.20 & 1.10 & 1.88 & 0.58 & $d, e$ \\
\hline $\mathrm{V}_{3} \mathrm{Ga}$ & $7.1^{*}$ & 0.62 & 0.20 & 1.23 & $2.9 f$ & 0.42 & $h$ \\
\hline
\end{tabular}

- Density of states for $\beta$-Wolfram type-compounds is taken as the number of states per eV per transition metal atom.

a This investigation.

b T.H. GEBALLE, B. T. MATTHIAS, J.P. MAITA, V.B. COMPTON, K. ANDRES and E. CORENZWIT, Bull. Am. Phys. Soc. 10, 579 (1965). Value of $\theta_{D}$ from J.P. MAITA, private communication.

c J.P. MAITA, private communication.

$d$ Value of $\theta_{D}$ from average of the velocity of sound. L.R. TESTARDI, private communication.

e J. E. KUNZLER, J.P. MAITA, H. J. LEVINSTeIN and E.J. RYDER, Phys. Rev., to be published.

$f$ The value of $N(0)$ calculated for $V_{3} G a$ can be compared in an interesting way with previous studies of this compound. Using values of the spin susceptibility determined by Knightshift experiments, a nonrenormalized density of states for $V_{3} G a$ equal to 3.1 states/eV/ atom was calculated. [A.M. CLOGSTON, Phys. Rev. 136, A8, 1964.] This calculation required an estimate of the exchange parameter $J$ and can only be considered a rough value for $N(0)$. It is nevertheless remarkably close to the value for $N(0)$ determined from $T_{c}$ and the specific heat. 


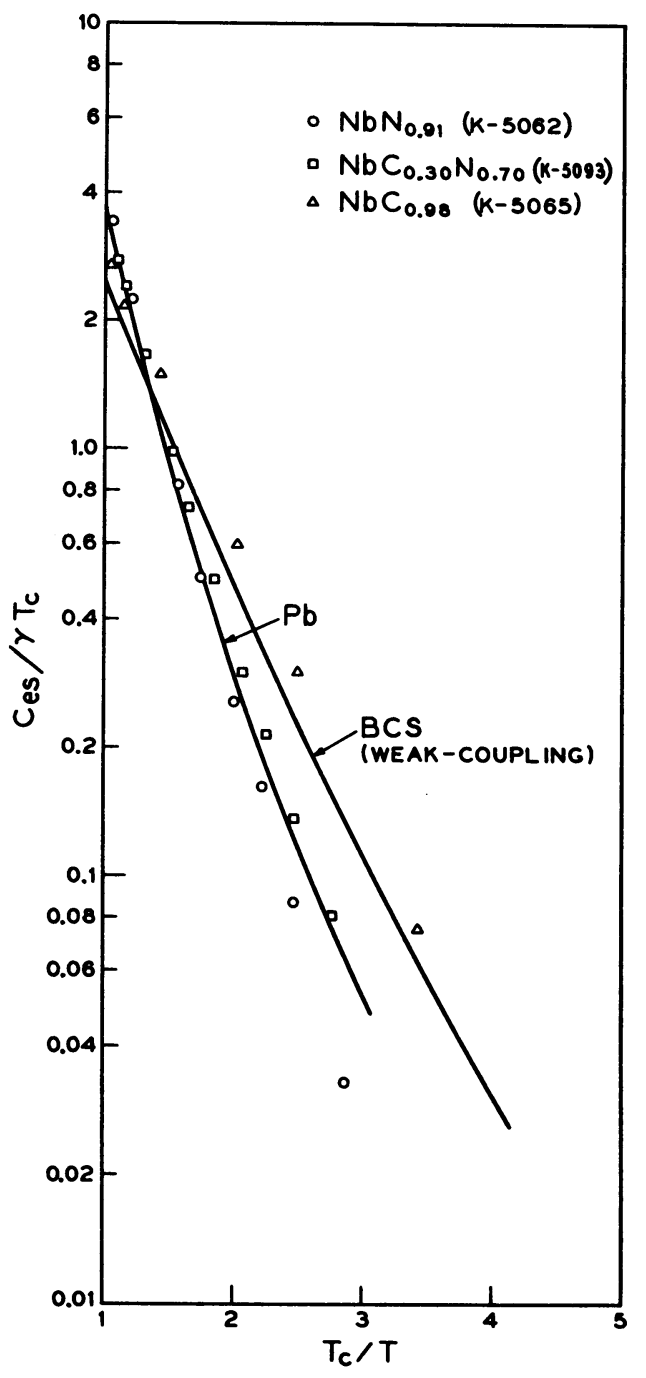

FIGURE 11

Normalized value of the electronic heat capacity in the superconducting state.

The solid curves represent values calculated for the BCS weak-coupling case (see footnote 13) and the values measured for Pb [D.L. DECKER, D. E.MAPOTHER and R. W. SHAW, Phys. Rev. 112, 1888, 1958]. The points are calculated from the data shown in Figs. 1, 2, and 3.

$N(0) V_{p h}$. The estimated value of 0.2 is obtained from the following considerations. The pseudopotential is given by [22]

$$
N(0) U_{c}=\frac{N(0) V}{1+N(0) V \ln E_{f} / E_{c}}
$$

where $E_{f}$ is the Fermi energy and $E_{c}$ the cutoff energy of the phonon spectrum. $V$ is the screened Coulomb interaction and for free electrons is given approximately by 


$$
N(0) V=\frac{1}{2}\left(\frac{\sigma}{2 k_{f}}\right)^{2} \ln \left[1+\left(\frac{2 k_{f}}{\sigma}\right)^{2}\right]
$$

where $\sigma$ is the Thomas-Fermi screening constant. $N(0) V$ is rather insensitive to $\sigma$ and varies from $N(0) V=0.35$ for $\left(\sigma / 2 k_{f}\right)=1$ to $N(0) V=0.50$ for $\left(\sigma / 2 k_{f}\right)=\infty$. A reasonable universal value for $N(0) V$ is 0.4 . The sensitivity of $N(0) U_{c}$ to the choice of $N(0) V$ is also reduced because $N(0) V \ln \left(E_{f} / E_{c}\right)$ is a number of order 1 or 2 . For $\mathrm{V}_{3} \mathrm{Si}$ the band density of states is $N(0)=3$ states/ev/atom. We should use for $E_{f}$ a value of order the reciprocal of $N(0)$ or about $0.3 \mathrm{~V}$. If we choose a cutoff $E_{c}$ at about $0.03 \mathrm{~V}, \ln \left(E_{f} / E_{c}\right) \simeq 2.3$ and $N(0) U_{c} \simeq 0.20$.

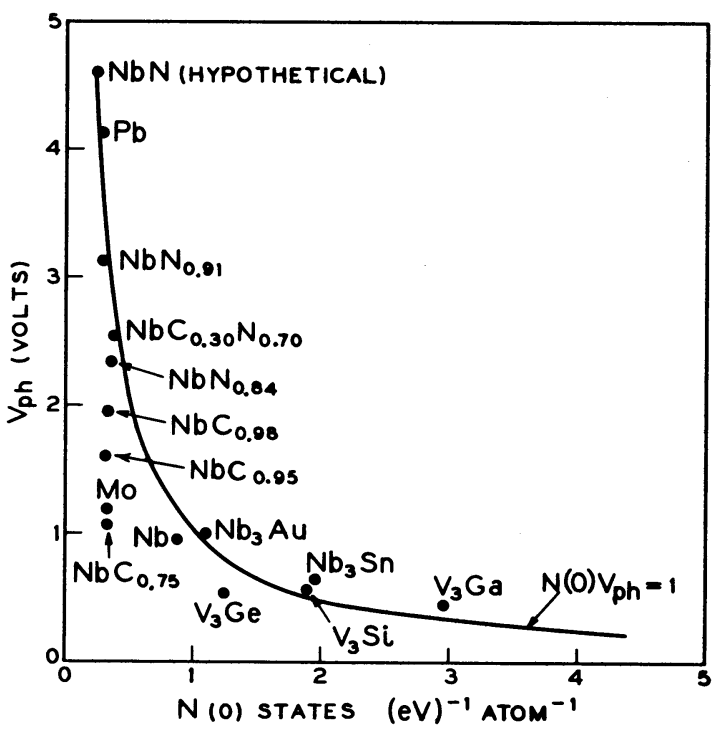

FIGURE 12

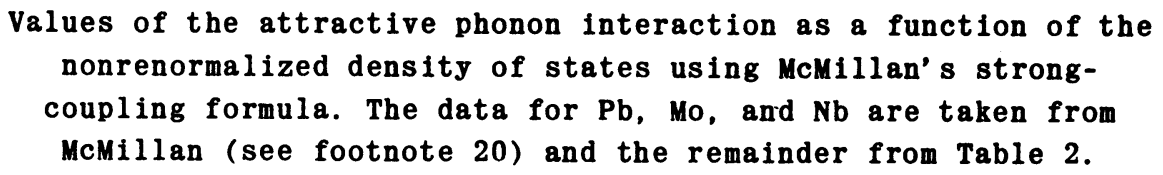

In Fig. 12, $V_{p h}$ is plotted as a function of $N(0)$. The curve $N(0) V_{p h}=1$ shown has no obvious theoretical significance but empirically it seems to represent and approximate boundary for superconductors. As can be seen, the high-temperature superconductors fall into two distinct groups - those with high $V_{p h}$ and low $N(0)$ and those with high $N(0)$ and low $V_{p h}$. The high $V_{p h}$ and low $N(0)$ values of $\mathrm{NbN}_{0.91}$ indicate that it resembles lead rather than $\mathrm{V}_{3} \mathrm{Si}$ or $\mathrm{V}_{3} \mathrm{Ga}$ although its Debye temperature is almost three times that of lead. Thus, insofar as its superconducting properties are concerned, $\mathrm{NbN}_{0.91}$ may be considered as a sort of "stiff lead". The increase in the superconducting transition temperature from $15.72^{\circ} \mathrm{K}$ for $\mathrm{NbN}_{0.91}$ to $17.38^{\circ} \mathrm{K}$ for $\mathrm{NbC}_{0.30} \mathrm{~N}_{0.70}$ as carbon is substituted for the nitrogen can be accounted for by the larger Debye temperature of $\mathrm{NbC}_{0.30} \mathrm{~N}_{0.70}$. The $N(0) V_{p h}$ product remains constant.

The $N(0) V_{p h}=1$ boundary of Fig. 12 could represent a limit imposed by lattice stability. A martensitic instability of $\mathrm{V}_{3} \mathrm{Si}$ has been reported [23]. The susceptibility curves of $\mathrm{NbN}_{0.91}$ and $\mathrm{NbC}_{0.30} \mathrm{~N}_{0.70}$ suggest that these materials may undergo a similar phase transition. $\mathrm{NbC}_{0.98}$ is well below the $N(0) V_{p h}=1$ boundary and shows normal susceptibility and heat capacity behavior. 


\section{(b) Proposed band structure}

An interpretation of the properties of the niobium interstitial compounds must take account of the observed susceptibilities, Knight shifts and specific heats. For $\mathrm{NbN}_{0.91}$ these measurements give (1) a susceptibility $X_{\text {meas }}=31.7 \times 10^{-6} \mathrm{e.m.u.} / \mathrm{mole}$ at room temperature (Fig. 7 ), (2) a Knight shift $k=0.02$ at room temperature [17], and (3) an unrenormalized density of states deduced from the specific heat equal to 0.29 states/eV/molecule (Table 2). The susceptibility $X_{\text {meas }}$ is very low for a transition metal compound. It would ordinarily be about ten times larger for the usual $d$-band widths. Taking account of a diamagnetic susceptibility $X_{d i a}$ of the niobium cores equal to about $20, \times 10^{-6} \mathrm{e} . \mathrm{m} . \mathrm{u} . / \mathrm{mole}$, the resulting positive susceptibility $X_{\text {meas }}-X_{d i a} \simeq 50 \times 10^{-6}$ could be accounted for by the Van vleck orbital susceptibility, $X_{v v}(4 d)$, of only $1 / 3$ to $1 / 24 d$ electrons assoctiated with the niobium atoms. This is an upper limit which makes no allowance for any Pauli spin susceptibility, $x_{p}(4 d)$, connected with the $d$ band.

Accordingly we would like to suggest the following picture for the bank structure of NbN (if it were stoichiometric). Lying lowest in energy there will be a 1-fold band composed essentially of nitrogen $2 s$ wave functions and filled with two electrons per molecule of $\mathrm{NbN}$. Next highest in energy will be a 3 -fold band strongly hybridized between the niobium $4 d$ wave functions and nitrogen $2 p$ wave functions. This bonding band will contain six electrons per molecule; it will contribute roughly three electrons to the niobium cell and three electrons to the nitrogen cell. At still higher energy there will be a plane wave band formed from niobium $5 s-5 p$ wave functions and nitrogen $3 s-3 p$ wave functions. The Fermi level will be high enough in this band so that it contains about two electrons per molecule. Above the Fermi level finally there will be a 5-fold anti-bonding band hybridized from niobium $4 d$ wave functions and nitrogen $2 p$ wave functions. This band will resemble the $4 d$ bands of the transition metals but will be almost completely unoccupied. The total average configuration of the niobium atoms will be $4 d^{3} 5 s$ and the nitrogen atoms $2 s^{2} 2 p^{3} 3 s$

This general picture of the band structure of $\mathrm{NbN}$ is similar to the model proposed by Bilz [24] and to the band structure calculated by Ern and Switendick [25] for Tio. Our major new suggestion is that in $\mathrm{NbN}$ the $4 d$ and $2 p$ hybridization is so strong that the anti-bonding $d$-like band has been pushed above the Fermi level which now lies in a plane wave conduction band.

On this model the conduction band would contain about 2 electrons/molecule of NbN. From the $X$-ray density of $8.36 \mathrm{~g} / \mathrm{cm}^{3}$ we can easily calculate a conduction electron (Pauli) spin susceptibility $X_{p}$ (cond) equal to approximately $13 \times 10^{-6} \mathrm{e} . \mathrm{m} . \mathrm{u} . / \mathrm{mole}$. The total susceptibility $X_{\mathrm{meas}}=$ $x_{d i a}+x_{p}($ cond $)+x_{p}(4 d)+x_{v v}(4 d)$ would now be made up of a contribution from $x_{d i a} \simeq-20 \times 10^{-6}$ e.m.u./mole, a contribution from $x_{p}$ (cond) $\simeq 13 \times 10^{-6}$ e.m.u./mole plus a total contribution from the $4 d$-like band that cannot exceed roughly $40 \times 10^{-6} \mathrm{e} . \mathrm{m} . \mathrm{u} . / \mathrm{g}$. This emphasizes once more the small extent to which the anti-bonding $d$-like band can be involved in the electronic structure.

We have a further check on this schematic band structure. The unrenormalized state density of 0.29 states/eV/molecule corresponds to a Pauli susceptibility (unenhanced by exchange) of $19 \times 10^{-6} \mathrm{e} . \mathrm{m} . \mathrm{u} . / \mathrm{mole}$. Subtracting the conduction band contribution of $13 \times 10^{-6} \mathrm{e} . \mathrm{m} . \mathrm{u} . / \mathrm{mole}$, this would leave a maximum value for the Pauli susceptibility due to the $4 d$-like band of $6 \times 10^{-6}$ e.m.u./mole. If the effective mass of these electrons is $m_{d}$, we have

$$
\mathrm{X}_{p}(4 d) / \mathrm{X}_{p}(\text { cond })=\left(m_{d} / m\right)\left(n_{d} / n_{c}\right)^{1 / 3}
$$

where the conduction electrons have mass $m$ and $n_{d}$ and $n_{c}$ are the number of carriers in the $4 d-$ like band and conduction band respectively. Using the above figures for $x_{p}(4 d)$ and $x_{p}$ (cond), we 
have $n_{d}=n_{c}\left(6 / 13 \mathrm{~m} / \mathrm{m}_{d}\right)^{3}$. This implies once more that $n_{d}$ must be very much less than 1 electron.

In terms of the present picture, the Knight shift results can be understood as follows: the conduction band susceptibility would produce a knight shift of the niobium nucleus of a few tenths per cent positive. A small additional positive term from the orbital susceptibility, and a small additional negative term from the Pauli susceptibility would arise from the $4 d$-like band. These three effects must add to give the very small observed Knight shift.

Many of the properties of the other niobium interstitial compounds listed in Table 1 can be understood qualitatively on the basis of the above model. Non-ideal stoichiometry, particularly vacancies in the sublattice of nitrogen atoms would be expected to cause the Fermi level to rise because the nitrogen atoms are negatively charged by one unit. Thus, $N(0)$ for $\mathrm{NbN}_{0.84}$ is greater than for $\mathrm{NbN}_{0.91}$. (Table 2) and hypothetical stoichiometric $\mathrm{NbN}$ is estimated to have an even lower $N(0)$. Equal numbers of vacancies in both sublattices, which according to Denker [26] occur to an appreciable extent in related structures, would be expected to have little effect on the position of the Fermi level. The Fermi level of niobium carbide must lie below niobium nitride due to the fewer number of electrons, and therefore the total contribution from the $4 d$-like band to the susceptibility should be less. Comparison of Figs. 7 and 9 shows that the susceptibility of niobium carbide is roughly one half that of niobium nitride. The observation that chromium in dilute solution in niobium nitride has a paramagnetic moment of $1.65 \mu_{B}$ and also strongly depresses the superconducting transition temperature is in agreement with the reported result that chromium in dilute solution in nontransition elements is often magnetized whereas in transition metals, chromium is not found to be magnetic $[27,28]$. In accordance with the present scheme the conduction band would run through $4 \mathrm{~s}$ wave functions of $\mathrm{Cr}$ atoms substituted for $\mathrm{Nb}$ and experience an exchange interaction with the locally magnetized $3 d$ wave functions. The usual effect of magnetized impurities on superconductivity should therefore be observed.

\section{(c) Maximum superconducting transition temperature}

Empirical evidence that there is an upper limit of $18^{\circ} \mathrm{K}$ for the occurrence of superconductivity has existed for some time $[19,29]$. The evidence was based on studies of $d$-band superconductors. The extensive work of Pessall and and Hulm [18] together with the present study

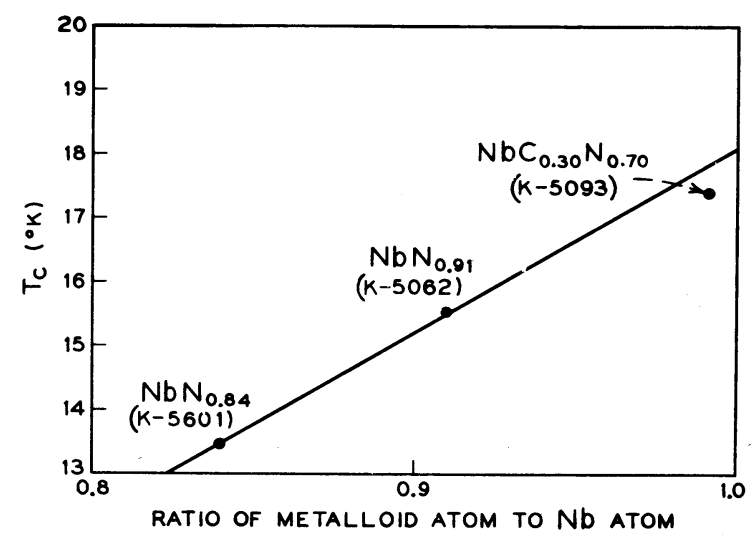

FIGURE 13

The transition temperature as a function of stoichiometry. The point for

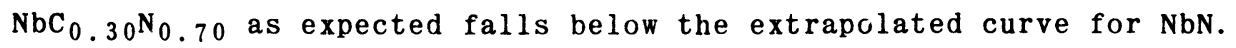

The substitution of carbon for nitrogen at constant stoichiometry lowers $T_{c}$. 
indicates that the $18^{\circ} \mathrm{K}$ limit applies to $s p$ band superconductors as well. It is interesting to speculate whether the transition temperature of a stoichiometric Bl-type niobium nitride, $\mathrm{NbN}$, if it existed, would be above $18^{\circ} \mathrm{K}$. An extrapolation of the transition temperature as a function of composition, as shown in Fig. 13, again points to an $18^{\circ} \mathrm{K}$ limit. The suggestion of a low-temperature transition which is seen in the susceptibility measurements of $\mathrm{NbN}_{0.91}$ and $\mathrm{NbC}_{0.30} \mathrm{~N}_{0.70}$ and the previously demonstrated low temperature transition in $\mathrm{V}_{3} \mathrm{Si}$ single crystals [23] lead to the speculation that perhaps the interactions strong enough to cause superconductivity to occur above $18^{\circ} \mathrm{K}$ may also be strong enough to cause lattice instabilities.

\section{Acknowledgments}

We are grateful to G.W. Hull, Jr. for his excellent work in measuring the superconducting transition temperatures and to E.M. Kelly and L.D. Longinotti for their careful preparation of many of the samples. We should like to thank $E$. Corenzwit for the arc-melted carbon-deficient sample and R.C. Sherwood for his help in the susceptibility measurements. The comments of $V$. Jaccarino regarding the Knight shift work were valuable. We have benefited from many helpful discussions with W.L. McMillan concerning the effects of strong-phonon coupling and with P.W. Anderson and V. Heine concerning possible band structures.

\section{References}

1. T. MEISSNER and H. FRANZ, Z. Phys, 65, 30 (1930).

2. G. ASCHERMANN, E. FRIEDERICH, E. JUSTI and J. KRAMER, Z. Phys. 42, 349 (1941).

3. A.L. GIORGI, E.G. SZKLARZ, E.K. STORMS, A.L. BOWMAN and B.T. MATthiAs, Phys. Rev. 125, 837 (1962).

4. H. ROGENER, Z. Phys, 132, 446 (1952).

5. B. T. MATthiAS, Phys. Rev. 92, 874 (1953).

6. E.K. STORMS and N.H. KRIKORIAN, J. Phys. Chem. 63, 1747 (1959).

7. A.L. SCHAWLOW and G.E. DEVLIN, Phys, Rev. 113, 120 (1959).

8. T. H. GEballe, B. T. matThias, V. B. COMPtON, E. CORENZWit, G. W. hULl Jr. and L. D. LONGINOTTI, Phys. Rev. 137, A119 (1965).

Q. F.J. MORIN and J.P. MAITA, Phys. Rev. 129, 1115 (1963).

10. R.M. BOZORTH, H.J. WILlitamS and D.E. WALSH, Phys. Rev. 103, 572 (1956).

11. G. T. ARmStrong, J. Am. Chem. Soc. 71, 3583 (1949).

12. T.A. SANDENAW and E.K. STORMS, Los Alamos Scientific Laboratory Report La 3331; J. Phys. Chem. Solids 27, 217 (1966).

13. J. BARDEEN and J.R. SCHRIEFFER, Recent developments in superconductivity, Progress in Low Temperature Physics (edited by C.J. Gorter), Vol. 3, p.170. North Holland, Amsterdam, (1961). 
14. H. BITTNER, H. GORETZKI, F. BENESOVSKY and H. NOWOTNY, Monatsh. Chem. 94, 518 (1963).

15. H. BITTNER and H. GORETZKI, Monatsh. Chem. 93, 1000 (1962).

16. We are indebted to $\mathrm{H}$. Levinstein for the low-temperature $\mathrm{X}$-ray diffraction investigation.

17. V. JACCARINO and J. DAVIS, private communication.

18. N. PESSALL and J.K. HULM, Physics 2, 311 (1966).

19. B. W. ROBERTS, Superconductive materials and some of their properties. Progress in Cryogenics (edited by K. Mendelsshohn), Vol. 4, p.160. Academic Press (1964).

20. W. L. McMILlaN, Personal communication, to be published.

21. A.B. MIGDAL, Soviet Phys.-JETP 7, 996 (1958).

22. N. N. BOGOLIUBOV, V.V. TOLMACHEV and D.V. SHIRKOV, A New Method in the Theory of Superconductivity. Consultants Bureau (1959).

23. B. W. BATTERMAN and C.S. BARRETT, Phys. Rev. Letters 13, 390 (1964); L.R. TESTARDI, T.B. BATEMAN, W. A. REED and V.G. CHIRBA, Phys. Rev. Letters 15, 250 (1965).

24. H. BILZ, Z. Phys. 153, 338 (1958).

25. V. ERN and A.C. SWITENDICK, Phys, Rev. 137, A1927 (1965).

26. S. P. DENKER, J. Phys. Chem. Solids 25, 1397 (1964).

27. F. J. du CHATENIER, Thesis, University of Leiden (1964).

28. G.J. van den BERG, Anomalies in dilute metallic solutions of transition metals, Low Temperature Physics LT9 (edited by J.G. Daunt, D. O. Edwards, F. J. Milford and M. Yaqub), p. 955. Plenum Press (1965).

29. B. T. MATthias, Rev. Mod. Phys. 33, 449 (1961); T. H. GEBALle and B. T. MatThias, Ann. Rev. Phys. Chem. 14, 141 (1963). 